\title{
A iconicidade como uma característica universal das línguas ${ }^{1}$
}

\author{
Daltro Roque Carvalho da SILVA-JÚNIOR @ \\ Universidade Federal do Paraná (UFPR) \\ André Nogueira XAVIER (D) \\ Universidade Federal do Paraná (UFPR)
}

\section{○}

OPEN ACCESS

EDITADO POR

- Raquel Freitag (UFS)

AVALIADO POR

- Anderson Almeida (UFPI)

SOBRE OS AUTORES

- Daltro R. C. da Silva-Júnior

Escrita - análise e edição.

- André Nogueira Xavier

Escrita - análise e edição.

DATAS

- Recebido: 19/07/2021

- Aceito: 09/09/2021

- Publicado: 24/09/2021

\section{COMO CITAR}

Silva-Júnior, D. R. C.; Xavier, A. N. (2021). A iconicidade como uma característica universal das línguas. Revista da Abralin, v. 20, n. 2, p. 1-10, 2021.

\section{RESUMO}

A apresentação do Prof. Bodo Winter tratou da iconicidade linguística. Primeiramente, ele mostrou que a iconicidade pode desempenhar diferentes funções na língua e que ela está presente em diferentes domínios linguísticos, entre eles o léxico. Como evidência disso, ele apresenta pesquisas que evidenciam a ocorrência de iconicidade no vocabulário relativo a tamanho, à forma e à aspereza do inglês. Ao final de sua apresentação e à luz dos trabalhos que a embasaram, o Prof. Bodo define a iconicidade como um processo seletivo (já que apenas alguns aspectos do significado podem ser representados depictivamente e não sua totalidade), que manifesta uma sensação de semelhança (portanto, interpretativo) e que depende do contexto em que ocorre para ser reconhecida. Ele ainda defende que a iconicidade seja considerada uma das características universais das línguas e não, como Saussure (1916) e Hockett (1960), a arbitrariedade, a qual considera epifenomenal.

\section{ABSTRACT}

Dr. Bodo Winter's presentation discussed linguistic iconicity. First, he showed that iconicity can perform different functions in language and that it is present in different linguistic domains among which is the lexicon. As evidence of that, he presents research that shows the occurrence of iconicity

\footnotetext{
${ }^{1}$ A versão em Libras desta resenha pode ser encontrada em: https://www.youtube.com/watch?v=-6ozshSO2C8
} 


\section{REVISTA DA ABRALIN}

in English vocabulary related to size, shape and roughness. At the end of his presentation and in light of the works on which it was based, Dr. Winter defines iconicity as a selective process (since only some aspects of meaning, not its entirety, can be depictively represented), that manifests a sense of resemblance (thus, interpretive), and that depends on the context in which it occurs to be recognized as such. He also claims that iconicity be considered one of the design features of languages and not, like Saussure (1916) and Hockett (1960), arbitrariness, which he considers to be epiphenomenal.

\section{PALAVRAS-CHAVE}

Iconicidade. Léxico. Universal linguístico.

\section{KEYWORDS}

Iconicity. Lexicon. Linguistic Universal.

A palestra do Prof. Bodo Winter objetiva demonstrar que a iconicidade e não a arbitrariedade se constitui com um traço arquitetônico, logo, universal, das línguas naturais. Para isso, ele organiza sua exposição em basicamente três grandes partes. Na primeira, ele reporta uma vasta literatura que evidencia não apenas que a iconicidade desempenha diferentes funções na língua, mas também sua pervasividade em diferentes domínios dela, entre elas no léxico. A segunda parte é dedicada a explorar a presença da iconicidade no léxico do inglês, domínio, em geral, negligenciado pela pesquisa linguística. Na terceira e última parte, o Prof. Bodo retoma a questão da iconicidade como um traço arquitetônico e propõe uma definição que engloba os vários aspectos que ela envolve. Neste texto, objetivamos sumarizar os principais pontos discutidos ao longo da palestra e destacar sua sólida fundamentação teórica e empírica, evidenciada pela vasta literatura citada ${ }^{2}$.

A primeira parte se inicia com o Prof. Bodo lembrando que a visão de língua tem se expandido e que atualmente ela inclui os gestos que coocorrem com a fala. Com isso, a língua passou a ser vista como multimodal, ou seja, como um fenômeno que, no caso das línguas orais, não se restringe à modalidade oro-auditiva, mas abrange também a modalidade visual-gestual ${ }^{3}$ (ENFIELD, 2009; PERNISS, 2018). O Prof. Bodo ressalta ainda que, além de multimodais, as línguas são também multissemióticas, pois seu uso envolve diferentes estratégias para criar significado, a saber, a descrição, a indicação e a depicção (FERRARA; HODGE, 2018).

\footnotetext{
${ }^{2}$ Agradecemos ao Prof. Bodo Winter por muito gentilmente disponibilizar as referências completas dos trabalhos citados ao longo de sua apresentação.

${ }^{3}$ Mais para o final da palestra, a multimodalidade das línguas será retomada, quando o Prof. Bodo cita os trabalhos de Hassemer e Winter $(2016,2018)$ sobre a iconicidade em configurações de mão de gestos que co-ocorrem com a fala.
} 


\section{REVISTA DA ABRALIN}

O Prof. Bodo explica que por meio da descrição identifica-se uma determinada entidade como membro de uma categoria mais geral e abstrata e a exemplifica com o enunciado "Isto é uma planta". Já a indicação se dá através de um apontamento na direção daquela planta. O Prof. Bodo explica que por meio dessa estratégia direciona-se a atenção dos interlocutores para uma determinada entidade. Por fim, ele descreve a depicção, que exemplifica com um gesto que se pode fazer em referência ao tamanho de uma planta. Ele ressalta, no entanto, que essas estratégias podem ser usadas separada ou conjuntamente.

A cada estratégia vincula-se um tipo de signo, respectivamente, arbitrário, indexical e icônico. Signos arbitrários, como a palavra tree 'árvore' do inglês apresentam uma forma que não representa depictivamente aspectos do seu significado. De acordo com o Prof. Bodo, isso fica ainda mais evidente quando essa palavra é comparada com a palavra correspondente em holandês baum. Vê-se que cada língua selecionou sons completamente diferentes para designar o mesmo referente. Signos indexicais, por sua vez, corresponderiam a gestos de apontamento, enquanto signos icônicos correspondem àqueles cuja forma se assemelha ao seu significado. Esse último tipo de signo é ilustrado por meio da palavra cukoo do inglês e da palavra TREE 'árvore' da língua de sinais americana, ASL. Ambas as palavras são icônicas, porque representam depictivamente em sua forma aspectos de seu significado, respectivamente, a vocalização do pássaro e a forma da árvore.

Baseado em pesquisas em diferentes frentes, o Prof. Bodo demonstra que a iconicidade está longe de ser uma característica marginal das línguas e que desempenha nelas diferentes funções. A iconicidade parece, por exemplo, facilitar a aquisição da linguagem (IMAI et al., 2008). Além disso, ela deve ter desempenhado um papel fundamental na origem da língua, quando ainda não havia uma convenção. Ele evidencia isso reportando estudos de laboratório em que sujeitos, ao serem impedidos de usar uma língua para se comunicar, são observados usando gestos e vocalizações icônicas para esse fim (SILVA et al., 2020). Nessa mesma linha, outros estudos demonstram que falantes e sinalizantes recorrem a formas mais icônicas, quando são solicitados a diferenciar entidades muito parecidas (LU; GOLDIN-MEADOW, 2018). A iconicidade é vastamente observada em usos lúdicos da língua, como jogos de palavras ou mesmo narrativas (DINGEMANSE; THOMPSON, 2020).

Somando-se às diferentes funções, o Prof. Bodo lista um considerável número de domínios da língua onde se pode observar a ocorrência de iconicidade, a saber, nas onomatopeias (PRATHA et al., 2016) que, em alguns casos, se lexicalizam como nomes de animais (BERLIN, 1992). Nos nomes de criaturas (KAWAHARA et al., 2018) e de personagens ficcionais (ELSEN, 2017), bem como em apelidos (SHIH; RUDIN, 2020) e nomes de produtos (KLINK, 2000). A iconicidade está presente também na poesia (TSUR, 1992; AURACHER et al., 2010), na prosódia (SHINTEL; NUSBAUM, 2007; PERLMAN et al., 2015), nos gestos (McNEILL, 1992; KENDON, 2004), nas línguas de sinais (BELLUGI; KLIMA, 1975; EMMOREY, 2014), nos ideofones ${ }^{4}$ (DINGEMANSE, 2012; AKITA; PARDESHI, 2019) e ainda nos emojis (COHN et al., 2019; McCULLOCH, 2019).

\footnotetext{
${ }^{4}$ Segundo o Prof. Bodo, são palavras empregadas para depicção sensorial. Como exemplo, ele cita o ideofone do japonês goro-goro, usado na depicção do movimento de rolar de uma entidade pesada. Ainda de acordo com ele, além do japonês, que exibe mais de
} 


\section{REVISTA DA ABRALIN}

Apesar de esses variados aspectos contarem com muitos estudos, alguns, inclusive, com uma vasta tradição, o Prof. Bodo menciona que são poucos os trabalhos que olham especificamente para a presença da iconicidade no léxico das línguas e, a propósito, introduz a pergunta que norteará a segunda parte de sua apresentação: “Há iconicidade no vocabulário geral do inglês?". Ele inicia essa discussão distinguindo a sistematicidade, relacionada a pareamentos recorrentes de forma e sentido no léxico, da iconicidade, embora ressalte haver entre ambos pontos de intersecção. Para ilustrar essa diferença, ele cita dois fonestemas ${ }^{5}$ do inglês: [gl] e [nk]. Segundo ele, ambos ocorrem sistematicamente no léxico do inglês em palavras que se referem à luminosidade (e.g.: glitter 'brilho', glitz 'brilho', glisten 'reluzir', gleam 'cintilar') e a um movimento abrupto (e.g.: clonk, clank, clink, boink), respectivamente. Entretanto, apenas o segundo fonestema, segundo ele, é iconicamente motivado. O Prof. Bodo embasa essa asserção na ocorrência translinguística da associação entre [nk] e a ideia de movimento abrupto.

Na sequência, casos semelhantes a este último são apresentados em outros domínios do léxico do inglês, a saber, 'tamanho', 'forma' e 'aspereza'. No que diz respeito ao vocabulário relacionado a tamanho, o Prof. Bodo, primeiramente, cita um estudo realizado por Sapir (1929) no qual solicitou-se a nativos de inglês e de chinês que escolhessem entre as pseudopalavras 'mil' e 'mal' a mais apropriada para nomear uma mesa pequena e uma mesa grande. Com base na escolha majoritária da palavra 'mil' para designar a mesa pequena e da palavra 'mal' para a mesa grande, o estudo concluiu que essa escolha foi motivada pela associação entre a compressão do trato vocal durante a articulação de [i] e o conceito 'pequeno', de um lado, e de sua expansão durante a produção de [a] e o conceito 'grande', de outro. Esse estudo foi criticado por Bentley e Varon (1933) por ter se baseado em pseudopalavras e consequentemente, na visão dos autores, por não revelar nada sobre o inglês especificamente que, inclusive, apresenta os adjetivos big 'grande' e small 'pequeno' como contra-exemplos à associação apontada pelo referido estudo. Em um estudo recente, Winter e Perlman (aceito) analisaram 18 guias de campo, que somam 6.000 nomes de pássaros referentes a aproximadamente 2.800 espécies. Desse total, os autores reportam que $70 \%$ são onomatopeias. Para testar se a forma da onomatopeia está correlacionada com o tamanho do pássaro, tais palavras foram categorizadas com base na sua constituição sonora e no tamanho do pássaro que nomeiam. Os autores observaram uma correlação muito alta entre a presença de alguns sons e o tamanho dos pássaros (r=0.67): [t] aparece frequentemente em nomes de pássaros pequenos e muito pouco frequentemente em pássaros grandes; [a] é muito frequente em nomes de pássaros grandes e ausente nos pequenos. Esse estudo contrasta com o de Katz (1986) que falhou em demonstrar iconicidade relacionada a tamanho no vocabulário do inglês. É provável que isso tenha ocorrido, segundo o Prof. Bodo, porque além de o estudo de Katz (1986) se concentrar apenas em vogais, diferentemente de Winter e Perlman (aceito), não focou em palavras especificamente relacionadas a tamanho. Replicando com sinônimos para

1000 ideofones, palavras desse tipo também são atestadas em línguas das Américas e da África. Línguas europeias não exibem ideofones.

${ }^{5}$ Grupos de fonemas específicos de uma língua que se parecem com morfemas, mas não apresentam composicionalidade (JOHANSSON et al., 2020). 


\section{REVISTA DA ABRALIN}

as palavras big 'grande' e small 'pequeno' do inglês, etimologicamente não relacionados, Winter e Perlman encontram alta correlação entre a constituição sonora dessas palavras e sua significação. [t] e [i] estão mais frequentemente presentes em adjetivos que significam 'pequeno', enquanto [a] foi mais frequentemente atestado em adjetivos que significam 'grande'. Dados translinguísticos indicam que essa sistematicidade é reflexo da iconicidade (BLASI et al., 2016).

Já em relação ao vocabulário relacionado à forma, o Prof. Bodo, primeiramente, sumarizou estudos que testaram a associação entre a forma de objetos e a configuração dos articuladores da fala. Esses estudos utilizaram duas imagens, uma com contornos pontiagudos e outra com contornos arredondados, e solicitaram que os sujeitos selecionassem uma pseudopalavra para nomeá-las: kiki/takete ou bouba/maluma (RAMACHANDRAN; HUBBARD, 2001; STYLES; GAWNE, 2017; FORT et al., 2018). Esses estudos indicaram uma tendência à associação de 'kiki/takete' à imagem pontiaguda e de 'bouba/maluma' à imagem de contornos arrendondados. Para verificar se a escolha da pseudopalavra kiki ou bouba não foi influenciada pelo formato das letras que as representam (observe-se que ' $k$ ' e 'i' são formas pontiagudas em comparação a 'b', 'o' e 'u', que apresentam formas arredondadas), Cuskley, Simner e Kirby (2017) replicaram esse mesmo estudo com 25 línguas de nove famílias diferentes e com diferentes sistemas de escrita. Embora os resultados tenham mostrado um efeito mais forte nas línguas que empregam o alfabeto romano, a tendência na escolha de 'kiki' para a imagem pontiaguda e de 'bouba' para a imagem sinuosa foi, de maneira geral, observada em todas as línguas. Sidhu et al. (2021) mostram em seu estudo que o efeito maluma/takete também é observado em nomes do inglês. Os autores reportam uma tendência na associação de palavras como spike 'espinho', fork 'garfo', cactus 'cacto' e shrapnel 'estilhaço' à imagem pontiaguada e de palavras como ball 'bola', globe 'globo', balloon 'balão' e hoop 'argola' à imagem de contorno arredondado.

Finalmente, a respeito do vocabulário relativo à aspereza, o Prof. Bodo mostrou a recorrência do rótico [r] e ilustrou isso através das palavras rugged 'áspero', rough 'duro', rocky 'rochoso', prickly 'espinhoso' e abrasive 'abrasivo'. Segundo ele, o uso desse som para sugerir aspereza é sistemático não só no inglês, mas também no húngaro. Para embasar que essa sistematicidade é reflexo da iconicidade, ele cita um levantamento que fez da palavra 'duro' em 329 línguas pertencentes a 83 famílias linguísticas diferentes. De acordo com ele, esse levantamento revelou que o uso do rótico foi prevalente em palavras correspondentes a ‘duro' e, em oposição, muito raro na palavra ‘suave'.

Após demonstrar convincentemente a presença de iconicidade no léxico do inglês, o Prof. Bodo passa à terceira e última parte de sua apresentação, na qual, primeiramente, contrapõe a visão saussureana, de acordo com a qual o signo linguístico é por natureza arbitrário, com a de linguistas como Bolinger e Sears (1981), para quem "basicamente tudo na língua tem uma origem não arbitrária". A partir disso, ele passa então a construir uma definição para a iconicidade que defende ser um dos traços arquitetônicos das línguas naturais. Para isso, primeiramente cita os trabalhos de Hassemer e Winter $(2016,2018)$, que mostram que configurações de mão usadas em gestos podem ser especificadas para tamanho e não para forma ou o contrário. Com base nisso, ele sustenta que a iconicidade é sempre seletiva, logo, nunca representa depictivamente todos os aspectos da entidade referida. Segundo o Prof. Bodo, a manifestação da seletividade é bastante clara em onomatopeias, uma vez 


\section{REVISTA DA ABRALIN}

que não conseguimos imitar, por exemplo, o cantar dos pássaros de maneira 100\% fiel. Ele chama a atenção ainda para o fato de que essa imitação passa por dois filtros: o da percepção auditiva humana e do da fonologia da língua do imitador. Segundo o Prof. Bodo, atribui-se ao segundo filtro a variação interlinguística em onomatopeias referentes à vocalização de um mesmo animal. Ele acredita, no entanto, que o papel desse filtro foi supervalorizado. Primeiramente porque um falante de inglês, por exemplo, poderia pronunciar onomatopeias de diferentes línguas. Em segundo lugar porque, como revelou o já referido estudo de Winter e Perlman (aceito), numa mesma língua podem-se encontrar diferentes onomatopeias para representar a vocalização correspondente a um mesmo animal. O Prof. Bodo atribui a existência de variação inter e intralinguística ao componente interpretativo da iconicidade. Em outras palavras, ele defende que as diferentes interpretações (ou seleções de aspectos perceptivamente salientes) gera variação, que nunca é arbitrária e da qual se selecionará a forma convencional.

Com base nisso, Prof. Bodo define a iconicidade como sendo um processo em que "aspectos da forma (linguística) exibem uma sensação de semelhança a aspectos do significado contextualmente instanciada". Em outras palavras, a iconicidade, além de seletiva, uma vez que apenas alguns aspectos do significado (e não sua totalidade) são representados, envolve uma sensação (interpretação) de semelhança (WILCOX, 2004; OCCHINO et al., 2017) e é contextualmente dependente (ORR, 1944). O Prof. Bodo ressalta, a propósito da dependência contextual, que não podemos predizer o significado a partir da forma, pois, de acordo com Orr (1944), "são as palavras e seu contexto que despertam as possibilidades expressivas latentes nos sons, e não os sons que conferem expressividade às palavras". De maneira mais expressiva, o Prof. Bodo diz que "a iconicidade não salta da palavra e nos dá o significado de graça". Para ilustrar isso, ele cita a vogal [i] que, fora de contexto, não significa nada. Segundo ele, apenas no âmbito de palavras como teeny 'pequenininho' é que ela evoca a ideia de tamanho e apenas no âmbito de palavras como gleam 'cintilar' que [gl] evoca a ideia de brilho. Essa possibilidade de um mesmo som, a depender do contexto, evocar ideias diferentes, é referida pelo Prof. Bodo como pluripotencialidade (WINTER, PÉREZ-SOBRINO, BROWN, 2019).

Conforme salienta o Prof. Bodo, a ubiquidade da iconicidade nas línguas, de uma forma geral, e no léxico, especificamente, contrasta com a visão consagrada encapsulada por Saussure (1916) em seu Princípio 1, de acordo com o qual o signo linguístico é arbitrário por natureza. O Prof. Bodo ainda chama a atenção para o fato que, a despeito da importância dada por Saussure à arbitrariedade, em sua obra não há uma definição clara do que ela é, mas apenas do que ela não é! Para o Prof. Bodo, a arbitrariedade do signo linguístico é epifenomenal, ou seja, emergente da análise das línguas de uma perspectiva comparativa. A seu ver, nesses casos, para que a iconicidade possa ser observada é preciso analisar a língua internamente ${ }^{6}$. $\mathrm{Na}$ mesma linha saussureana, lembra o Prof. Bodo, a

\footnotetext{
${ }^{6}$ De fato, comparando-se a palavra do inglês tree 'árvore' com a palavra correspondente em outras línguas, tem-se a impressão de que cada língua fez escolhas arbitrárias quando convencionam um determinado significante para representar um determinado significado. No entanto, como uma simples consulta a um dicionário etimológico pode revelar, a palavra tree descende da palavra reconstruída *deru- do PIE, língua na qual significava algo como "ser firme, sólido" (ONLINE ETYMOLOGY DICTIONARY, 2021). Com
} 


\section{REVISTA DA ABRALIN}

arbitrariedade figura como um dos 13 traços arquitetônicos das línguas humanas listados por Hockett (1960) ${ }^{7}$. Na visão do professor, no entanto, é a capacidade de estabelecer convenções, embutida no traço "Transmissão por tradição" que deveria ser considerada com um princípio universal, já que, por independer da existência de um link entre forma e sentido, torna a arbitrariedade possível.

O Prof. Bodo destaca que a arbitrariedade é epifenomenal também em virtude de poder resultar da ocorrência de processos linguísticos que, apesar de terem motivações próprias, ou seja, de não serem eles mesmos arbitrários, contribuem com a sua erosão. Entre esses processos ele cita aqueles que levam à redução (morfo)fonológica (PERLMAN et al., 2015; NAMBOODIRIPAD et al., 2016), a mudanças semânticas e fonológicas e mesmo a empréstimos linguísticos (FLAKSMAN, 2020). Para finalizar, o Prof. Bodo chama a atenção para o fato de que "(i) há muito mais iconicidade nas línguas do que tradicionalmente se reconhece; (ii) a iconicidade é "fluida" (interpretativa, contextualmente dependente, pluripotencial) e (iii) epifenomenal.

A nosso ver, a palestra do Prof. Bodo Winter representa uma valiosa contribuição para a linguística. Entre as muitas razões, destacamos a demonstração, com base em uma revisão de literatura de grande fôlego, da pervasividade da iconicidade nas línguas, inclusive no léxico, domínio em que ainda se sente forte influência da visão saussureana. Além disso, ao demonstrar que há muito mais iconicidade nas línguas faladas do que normalmente se assume, o professor contribui com uma mudança de visão sobre as línguas sinalizadas, que já tiveram seu estatuto de língua natural negado por serem relativamente mais icônicas e que ainda são vistas por muitos como exóticas pelo mesmo motivo.

\section{REFERÊNCIAS}

Akita, K.; Pardeshi, P. Ideophones, mimetics and expressives. John Benjamins, 2019.

Auracher, J.; Albers, S.; Zhai, Y.; Gareeva, G.; Stavniychuk, T. P is for happiness, N is for sadness: Universals in sound iconicity to detect emotions in poetry. Discourse Processes, 48(1), p. 1-25, 2010.

Bellugi, U.; Klima, E. S. Aspects of sign language and its structure. In J. Kavanagh \& J. Cutting (Org.). The role of speech in language, MIT Press, 1975, p. 171-203.

Bentley, M.; Varon, E. J. An accessory study of "phonetic symbolism". The American Journal of Psychology, 45(1), p. $76-86,1933$.

isso, fica claro que, apesar de, aparentemente, aspectos da forma da árvore não serem representados sonoramente, etimologicamente se vê uma clara motivação semântica para o uso dessa palavra para nomear a entidade em discussão.

\footnotetext{
${ }^{7}$ Vale registrar que em trabalho posterior, Hockett, ao reconhecer que as línguas de sinais são línguas naturais, relativiza a aparente arbitrariedade das línguas faladas (HOCKETT, 1978). Segundo ele, as línguas faladas são comparativamente às línguas sinalizadas menos icônicas por conta de sua modalidade de manifestação. Diferentemente das línguas de sinais, que, por serem tridimensionais como a própria vida, podem lançar mão de figuras feitas no ar, pantomimas e apontamentos, as línguas faladas, em sua visão, precisam comprimir essa tridimensionalidade em uma única dimensão e, em razão disso, suprimir a maior parte da iconicidade (p. 275).
} 


\section{REVISTA DA ABRALIN}

Berlin, B. Ethnobiological classification: Principles of categorization of plants and animals in traditional societies. Princeton University Press, 1992.

Blasi, D. E.; Wichmann, S.; Hammarström, H.; Stadler, P. F.; Christiansen, M. H. Sound-meaning association biases evidenced across thousands of languages. In: Proceedings of the National Academy of Sciences, 113(39), 1081810823, 2016.

Bolinger, D.; Sears, D. A. Aspects of language. New York: Harcourt Brace Jovanovich. Chicago, 1981.

Cohn, N.; Engelen, J.; Schilperoord, J. The grammar of emoji? Constraints on communicative pictorial sequencing. Cognitive Research: Principles and Implications, 4(1), p. 1-18, 2019.

Cuskley, C.; Simner, J.; Kirby, S. Phonological and orthographic influences in the bouba-kiki effect. Psychological Research, 81(1), p. 119-130, 2017.

Dingemanse, M.; Thompson, B. Playful iconicity: Structural markedness underlies the relation between funniness and iconicity. Language and Cognition, p. 1-22, 2020.

ELSEN, H. The two meanings of sound symbolism. Open Linguistics, 3(1), p. 491-499, 2017.

Dingemanse, M. Advances in the cross-linguistic study of ideophones. Language and Linguistics Compass, 6(10), p. 654-672, 2012.

Emmorey, K. Iconicity as structure mapping. Philosophical Transactions of the Royal Society B: Biological Sciences, 369(1651), 2014

ENFIELD, N. J. The anatomy of meaning: Speech, gesture, and composite utterances (Vol. 8). Cambridge University Press, 2009.

Etymonline.com. 2001-2021. Online Etymology Dictionary. [online] Disponível em: https://www.etymonline.com/ [Acessado 15 Jul 2021].

Ferrara, L.; Hodge, G. Language as description, indication, and depiction. Frontiers in Psychology, 9, 2018.

Flaksman, M. Iconic treadmill hypothesis. Dimensions of Iconicity. Iconicity in Language and Literature, 15, p. 15$38,2017$.

Flaksman, M. Pathways of de-iconization: How borrowing, semantic evolution, and regular sound changes obscure iconicity. Iconicity in Language and Literature, 17, p. 75-103, 2020.

Fort, M.; Lammertink, I.; Peperkamp, S.; Guevara-Rukoz, A.; Fikkert, P.; Tsuji, S. Symbouki: A meta-analysis on the emergence of sound symbolism in early language acquisition. Developmental Science, 21(5), 2018.

Hassemer, J.; Winter, B. Producing and perceiving gestures conveying height or shape. Gesture, 15(3), p. 404-424, 2016

Hassemer, J.; Winter, B. Decoding gestural iconicity. Cognitive Science, 42(8), p. 3034-3049, 2018.

HOCKETT, C. F. In Search of Jove's Brow. American Speech, v. 53, n. 4, p. 243-313, 1978.

Hockett, C. F. The origin of speech. Scientific American, 203(3), p. 88-97, 1960. 


\section{REVISTA DA ABRALIN}

ICONICITY, NOT ARBITRARINESS, IS A DESIGN FEATURE OF LANGUAGE. Conferência apresentada por Bodo Winter [s.l., s.n], 2021. 1 vídeo (1h 37min 47s). Publicado pelo canal da Associação Brasileira de Linguística. Disponível em: https://www.youtube.com/watch?v=R1ETw21oCGE\&t=1438s. Acesso em 16 jul, 2021.

Imai, M.; Kita, S.; Nagumo, M.; Okada, H. Sound symbolism facilitates early verb learning. Cognition, 109(1), p. 54$65,2008$.

KATZ, A. N. Meaning conveyed by vowels: Some reanalyses of word norm data. Bulletin of the Psychonomic Society, 24(1), p. 15-17, 1986.

Kawahara, S.; Noto, A.; Kumagai, G. Sound symbolic patterns in Pokémon names. Phonetica, 75(3), p. 219-244, 2018.

KENDON, A. Gesture: Visible action as utterance. Cambridge University Press, 2004.

KLINK, R. R. Creating brand names with meaning: The use of sound symbolism. Marketing Letters, 11(1), p. 5-20, 2000 .

Lu, J.; Goldin-Meadow, S. Combining categorical and gradient information in sign and spoken communication. In: 11th International Symposium on Iconicity in Language and Literature, Brighton, UK: University of Brighton, 6(8), 2017.

McCulloch, G. Because internet: Understanding how language is changing. Random House, 2019.

McNEILL, D. Hand and mind: What gestures reveal about thought. University of Chicago Press, 1992.

Namboodiripad, S.; Lenzen, D.; Lepic, R.; Verhoef, T. Measuring conventionalization in the manual modality. Journal of Language Evolution, 1(2), p. 109-118, 2016.

Occhino, C.; Anible, B.; Wilkinson, E.; Morford, J. P. Iconicity is in the eye of the beholder: How language experience affects perceived iconicity. Gesture, 16(1), 100-126, 2017.

ORR, J. On some sound values in English. British Journal of Psychology, 35(1), 1944.

Perlman, M.; Clark, N.; Johansson Falck, F. M. (2015). Iconic prosody in story reading. Cognitive Science, 39(6), p. 1348-1368.

Perlman, M.; Clark, N.; Johansson Falck, M. Iconic prosody in story reading. Cognitive Science, 39(6), p. 1348-1368, 2015.

Perlman, M.; Dale, R.; Lupyan, G. Iconicity can ground the creation of vocal symbols. Royal Society Open Science, 2(8), 150152, 2015.

Perniss, P. Why we should study multimodal language. Frontiers in Psychology, 9, 1109, 2018.

Pratha, N. K.; Avunjian, N.; Cohn, N. Pow, punch, pika, and chu: The structure of sound effects in genres of American comics and Japanese manga. Multimodal Communication, 5(2), p. 93-109, 2016.

Ramachandran, V. S.; Hubbard, E. M. Synaesthesia-a window into perception, thought and language. Journal of Consciousness Studies, 8(12), p. 3-34, 2001.

SAPIR, E. A study in phonetic symbolism. Journal of Experimental Psychology, 12(3), p. 225-239, 1929. 


\section{REVISTA DA ABRALIN}

Saussure, F.; Bally, C.; Sechehaye, A. Course in general linguistics. New York: McGraw-Hill, 1966.

Shih, S.; Rudin, D. On sound symbolism in baseball player names. Names, p. 1-18, 2020.

Shintel, H.; Nusbaum, H. C. The sound of motion in spoken language: Visual information conveyed by acoustic properties of speech. Cognition, 105(3), p. 681-690, 2007.

Sidhu, D. M.; Westbury, C.; Hollis, G.; Pexman, P. M. Sound symbolism shapes the English language: The maluma/takete effect in English nouns. Psychonomic Bulletin \& Review, p. 1-9, 2021.

Silva, M. V.; Holler, J.; Ozyurek, A.; Roberts, S. G. Multimodality and the origin of a novel communication system in face-to-face interaction. Royal Society Open Science, 7(1), 182056, 2020.

Styles, S. J.; Gawne, L. When does maluma/takete fail? Two key failures and a meta-analysis suggest that phonology and phonotactics matter. I-Perception, 8(4), $2041669517724807,2017$.

Tsur, R. What makes sound patterns expressive?: The poetic mode of speech perception. Duke University Press, 1992.

Wilcox, S. Cognitive iconicity: Conceptual spaces, meaning, and gesture in signed language. Cognitive Linguistics, 15(2), p. 119-147, 2004.

Winter, B.; Perlman, M. Size sound symbolism in the English lexicon. Glossa: a journal of general linguistics, 6(1), p.79, 2021.

Winter, B.; Pérez-Sobrino, P.; Brown, L. The sound of soft alcohol: Crossmodal associations between interjections and liquor. PLoS ONE, 14(8), 2019. 